\title{
Inflammation-based prognostic scores predict the prognosis of locally advanced cervical esophageal squamous cell carcinoma patients receiving curative concurrent chemoradiotherapy: A propensity score-matched analysis
}

Chia-Che Wu ${ }^{1}$, Shau-Hsuan Li ${ }^{1}$, Hung-I Lu ${ }^{2}$, Chien-Ming Lo ${ }^{2}$, Yu-Ming Wang ${ }^{3}$, Shang-Yu Chou ${ }^{3}$, YenHao Chen Corresp. 1,4,5

${ }^{1}$ Department of Hematology-Oncology, Kaohsiung Chang Gung Memorial Hospital, Kaohsiung, Kaohsiung, Taiwan

2 Department of Thoracic \& Cardiovascular Surgery, Kaohsiung Chang Gung Memorial Hospital, Kaohsiung, Kaohsiung, Taiwan

3 Department of Radiation Oncology, Kaohsiung Chang Gung Memorial Hospital, Kaohsiung, Kaohsiung, Taiwan

${ }^{4}$ Graduate Institute of Clinical Medical Sciences, College of Medicine, Chang Gung University, Taoyuan, Taoyuan, Taiwan

${ }^{5}$ School of Medicine, Chung Shan Medical University, Taichung, Taichung, Taiwan

Corresponding Author: Yen-Hao Chen

Email address: alex2999@cgmh.org.tw

Introduction: The present study investigated the crucial role of inflammation-based prognostic scores in locally advanced cervical esophageal squamous cell carcinoma (ESCC) patients who underwent curative concurrent chemoradiotherapy (CCRT).

Methods: There were 411 ESCC patients enrolled, including 63 cervical ESCC patients. Using the propensity score matching method, 63 thoracic ESCC patients were matched to the 63 cervical ESCC patients. The inflammation-based prognostic scores included the neutrophil lymphocyte ratio (NLR), platelet lymphocyte ratio (PLR), albumin level, c-reactive protein (CRP) level, modified Glasgow prognostic score (mGPS), and CRP/albumin ratio. The chi-square test and Kaplan-Meier method were used for categorical variable data and overall survival, respectively. A Cox regression model was performed for univariate and multivariable analyses.

Results: With respect to cervical ESCC, NLR $\geq 2.5(P=0.019), P L R \geq 103(P=0.013)$, CRP value $>10 \mathrm{mg} / \mathrm{l}$ $(P=0.040), m G P S \geq 1(P=0.040)$, and $C R P / a l b u m i n$ ratio $\geq 9.5(P=0.033)$ were significant predictors of worse overall survival (OS) in the univariate analysis. In a multivariable analysis, $P L R \geq 103(P=0.010$, HR: $2.66,95 \% \mathrm{Cl}: 1.27-5.58)$ and $\mathrm{mGPS} \geq 1$ ( $\mathrm{P}=0.030, \mathrm{HR}: 2.03,95 \% \mathrm{Cl}: 1.07-3.86)$ were the independent prognostic parameters of worse OS. The prognostic value of these biomarkers in the matched thoracic ESCC patients was similar and compatible with the results in the cervical ESCC group in the univariate and multivariable analyses.

Conclusions: Our study suggests that these inflammation-based prognostic scores are helpful in clinical practice, and PLR and mGPS may predict the prognosis for locally advanced cervical ESCC patients who receiving curative CCRT. 
1 Inflammation-based prognostic scores predict the prognosis of locally advanced cervical

2

5 Chia-Che $\mathrm{Wu}, \mathrm{MD}^{1 \#}$, Shau-Hsuan Li, MD ${ }^{1 \#}$, Hung-I Lu, MD ${ }^{2}$ Chien-Ming Lo, MD ${ }^{2}$

6 Yu-Ming Wang, MD, ${ }^{3}$ Shang-Yu Chou, MD, ${ }^{3}$ and Yen-Hao Chen, MD, $, 1,4,5^{*}$

7

8 Affiliations:

$9{ }^{1}$ Department of Hematology-Oncology, Kaohsiung Chang Gung Memorial Hospital and Chang

10 Gung University College of Medicine, Kaohsiung, Taiwan

$11{ }^{2}$ Department of Thoracic \& Cardiovascular Surgery, Kaohsiung Chang Gung Memorial Hospital

12 and Chang Gung University College of Medicine, Kaohsiung, Taiwan

$13{ }^{3}$ Department of Radiation Oncology, Kaohsiung Chang Gung Memorial Hospital and Chang

14 Gung University College of Medicine, Kaohsiung, Taiwan

$15{ }^{4}$ Graduate Institute of Clinical Medical Sciences, College of Medicine, Chang Gung University,

16 Taoyuan, Taiwan 
$17{ }^{5}$ School of Medicine, Chung Shan Medical University, Taichung, Taiwan

$19{ }^{\#}$ These authors contributed equally to the work

$21 *$ Correspondence: Yen-Hao Chen, MD, Department of Hematology-Oncology, Kaohsiung

22 Chang Gung Memorial Hospital and Chang Gung University College of Medicine, Kaohsiung,

23 Taiwan.

24 No.123, Dapi Rd., Niaosong Dist., Kaohsiung City 833, Taiwan (R.O.C.)

25 Tel: 886-7-7317123 ext.8303, Fax: 886-7-7322402,

26 E-mail: alex8701125@gmail.com 


\section{Abstract}

35 Introduction: The present study investigated the crucial role of inflammation-based prognostic

36 scores in locally advanced cervical esophageal squamous cell carcinoma (ESCC) patients who

37 underwent curative concurrent chemoradiotherapy (CCRT).

38 Methods: There were 411 ESCC patients enrolled, including 63 cervical ESCC patients. Using

39 the propensity score matching method, 63 thoracic ESCC patients were matched to the 63

40 cervical ESCC patients. The inflammation-based prognostic scores included the neutrophil

41 lymphocyte ratio (NLR), platelet lymphocyte ratio (PLR), albumin level, c-reactive protein (CRP)

42 level, modified Glasgow prognostic score (mGPS), and CRP/albumin ratio. The chi-square test

43 and Kaplan-Meier method were used for categorical variable data and overall survival,

44 respectively. A Cox regression model was performed for univariate and multivariable analyses.

45 Results: With respect to cervical ESCC, NLR $\geq 2.5(\mathrm{P}=0.019), \mathrm{PLR} \geq 103(\mathrm{P}=0.013), \mathrm{CRP}$

46 value $>10 \mathrm{mg} / 1(\mathrm{P}=0.040), \mathrm{mGPS} \geq 1(\mathrm{P}=0.040)$, and $\mathrm{CRP} /$ albumin ratio $\geq 9.5(\mathrm{P}=0.033)$ were

47 significant predictors of worse overall survival (OS) in the univariate analysis. In a multivariable

analysis, $\mathrm{PLR} \geq 103(\mathrm{P}=0.010, \mathrm{HR}: 2.66,95 \% \mathrm{CI}: 1.27-5.58)$ and $\mathrm{mGPS} \geq 1(\mathrm{P}=0.030, \mathrm{HR}: 2.03$, 
49 95\% CI: 1.07-3.86) were the independent prognostic parameters of worse OS. The prognostic

50 value of these biomarkers in the matched thoracic ESCC patients was similar and compatible

51 with the results in the cervical ESCC group in the univariate and multivariable analyses.

52 Conclusions: Our study suggests that these inflammation-based prognostic scores are helpful in

53 clinical practice, and PLR and mGPS may predict the prognosis for locally advanced cervical

54 ESCC patients who receiving curative CCRT.

\section{Introduction}

Esophageal cancer is one of the most fatal human malignancies worldwide. In Taiwan,

esophageal squamous cell carcinoma (ESCC) is the major pathologic type of esophageal cancer,

accounting for more than $90 \%$ of all cases, and is the ninth leading cause of cancer-related deaths

60 (National Department of Health, Republic of China, 2015). The cervical esophagus is a small

61 portion of the esophagus with a length of $5 \mathrm{~cm}$, and cervical ESCC accounts for only a small

62 portion, specifically, less than 10\%, of all esophageal cancer cases (Yin et al., 1983). In the past,

63 the standard treatment for cervical ESCC was radical surgery, radiotherapy, or a combination of

64 both. However, the surgery usually consisted of laryngoesophagectomy and reconstruction with 
65 gastric transposition or colon graft. Moreover, even with such surgery, the 5-year survival rate

66 was only $12 \%$ to $27 \%$ and the post-operative mortality rate was high at $6 \%$ to $20 \%$ with

67 significant morbidities (Grass et al., 2015). Recently, however, several studies have shown that

68 concurrent chemotherapy $(\mathrm{CCRT})$ improves survival rates for ESCC and head/neck cancer

69 patients; therefore, some physicians preferred definitive CCRT rather than surgical resection for

70 cervical ESCC patients in clinical practice, especially for locally advanced status (Cooper et al.,

71 1999; Pignon et al., 2009).

72 Growing evidences have revealed that inflammation plays an important role in tumor cell

73 proliferation, migration, invasion, and metastasis, as well as disease progression (Balkwill \&

74 Mantovani, 2001; Mantovani et al., 2008). A series of inflammatory biomarkers, such as the

75 neutrophil lymphocyte ratio (NLR), platelet lymphocyte ratio (PLR), albumin level, c-reactive

76 protein (CRP) level, modified Glasgow prognostic score (mGPS), and CRP/albumin ratio, have

77 been identified to predict clinical outcomes in several cancer types, including esophageal cancer

78 (Feng et al., 2014; Lindenmann et al., 2014; Pinato et al., 2014; Stotz et al., 2013; Templeton et

79 al., 2014; Yodying et al., 2016). 
80 With respect to esophageal cancer, these biomarkers were reported to be associated with

81 tumor progression and prognosis in esophageal cancer patients who receive different therapeutic

82 modalities, including surgical resection, neoadjuvant chemoradiotherapy followed by

83 esophagectomy, esophagectomy followed by adjuvant chemoradiotherapy, and definitive

84 chemoradiotherapy (Dutta et al., 2011; Feng et al., 2013; Miyata et al., 2011; Sharaiha et al.,

85 2011; Yoo et al., 2014). However, to the best of our knowledge, these biomarkers have not been

86 evaluated in cervical ESCC patients who receive curative CCRT.

87 In the present study, the locally advanced cervical ESCC patients who received curative

88 CCRT in our hospital were retrospectively reviewed, and the aim of the study was to determine

89 the clinical impact of inflammation-based prognostic scores in locally advanced cervical ESCC

90 patients who have undergone curative CCRT.

\section{Material and Methods}

\section{Patient population}

94 Study approval was obtained from the Chang Gung Medical Foundation Institutional

95 Review Board (201800845B0), and written informed consent from the patients or their families 
96 was not judged necessary for this kind of retrospective study. We retrospectively reviewed ESCC

97 patients with available medical records who underwent treatment between January 2005 and

98 December 2015 at Kaohsiung Chang Gung Memorial Hospital. The eligibility criteria were as

99 follows: (1) squamous cell carcinoma in histology (2) locally advanced status, stage III, without

100 distant metastasis or neck/celiac lymph node metastasis; (3) complete CCRT with curative intent;

101 (4) survive more than 3 months after completing CCRT; (5) no history of second primary

102 malignancy, such as head and neck cancers; (6) no form of any acute or chronic

103 infection/inflammatory disease; and (7) Eastern Cooperative Oncology Group performance

104 status 0-1. Ultimately, there were 411 ESCC patients who met the criteria for further analysis,

105 including 63 patients who had tumors located in the cervical esophagus and 348 patients with

106 thoracic esophageal tumors.

107 In order to prevent selection bias for better comparison, the propensity score matching

108 method was used among the 348 thoracic ESCC patients. First, we used binary logistic

109 regression to calculate a propensity score, with covariates including age, gender, tumor T status,

110 tumor $\mathrm{N}$ status, tumor stage, and tumor grade being entered into the propensity model. 
111 Subsequently, a 1-to-1 match with the closest matching scores between the 63 cervical thoracic

112 patients and 63 thoracic ESCC patients was determined. The algorithm used is shown in Figure 1.

114 Definition of inflammatory biomarkers and clinical tumor stage

115 In our study, chest computed tomography (CT), endoscopic ultrasonography (EUS), and

116 positron emission tomography (PET) scans were performed for each patient, and the clinical

117 tumor stage was determined according to the $7^{\text {th }}$ American Joint Committee on Cancer (AJCC)

118 staging system (Edge $\mathrm{S}$ et al., 2010). The definition of cervical esophageal cancer is that the

119 tumor lies in the neck and is bordered superiorly by the hypopharynx and inferiorly by the

120 thoracic inlet (sternal notch), approximately $15-20 \mathrm{~cm}$ from the incisors (Edge $\mathrm{S}$ et al., 2010).

121 Blood samples were obtained before treatment to measure the biomarkers of interest, which

122 included the white blood cell count, platelet count, neutrophil count, lymphocyte count, albumin

123 level, and CRP level. The NLR was calculated by dividing the neutrophil count by the

124 lymphocyte count, and the PLR was defined as the platelet count divided by the lymphocyte

125 count; the cut-off values for the NLR and PLR were 2.5 and 103, respectively (Dutta et al., 2011;

126 Xie et al., 2016). The cut-off levels for CRP and albumin in this study were $10 \mathrm{mg} / \mathrm{l}$ and $3.5 \mathrm{~g} / \mathrm{dl}$, 
127 respectively, with these levels being based on those used in previous studies (Forrest et al., 2003;

128 McMillan, 2008; McMillan, 2013; McMillan et al., 2001). The mGPS was calculated using the

129 CRP and albumin values and the scoring system was as follows: (1) patients with a normal CRP

130 value $(\leq 10 \mathrm{mg} / \mathrm{l})$ were allocated a score of 0 , regardless of the albumin level; (2) patients with a

131 CRP level $>10 \mathrm{mg} / 1$ combined with an albumin level $\geq 3.5 \mathrm{~g} / \mathrm{dl}$ were allocated a score of $1 ;$; (3)

132 patients with a CRP $>10 \mathrm{mg} / \mathrm{l}$ and an albumin $<3.5 \mathrm{~g} / \mathrm{dl}$ were allocated a score of 2 (McMillan,

133 2008). The optimal cut-off level for the CRP/albumin ratio was defined as 9.5 in the subsequent

134 analysis (Wei et al., 2015). All the indicators involved in the calculation of the inflammation-

135 based prognostic scores were examined before the patients underwent chemotherapy and

136 radiotherapy.

139 Curative intent radiotherapy was planned for each patient, and the details are described

140 below. First, a customized thermoplastic immobilization device was designed. Subsequently,

141 CT-simulation for image acquisition was performed. Inverse plan intensity-modulated

142 radiotherapy (IMRT) was then used to deliver 6- or 10-MV photons to cover the treatment field, 
143 including the neck and mediastinum. The gross target volume (GTV) was defined as the gross

144 tumor and gross lymph nodes (LNs) according to chest CT scan and/or PET-CT images. The

145 clinical target volume (CTV) comprehensively covered the esophagus, the mediastinal LNs, and

146 the supraclavicular LNs. The planning target volume (PTV) was defined as the CTV expanded

147 by $0.5-1.0 \mathrm{~cm}$ margins in all directions. The total doses prescribed to the PTV were $66-70 \mathrm{~Gy}$ in

148 33-35 daily fractions for cervical ESCC and 50-50.4 Gy in 25-28 daily fractions for thoracic

149 ESCC, followed by a boost dose to the gross neck LNs for an additional 10-16 Gy in 5-8 daily

150 fractions.

151 Chemotherapy was performed concurrently with radiotherapy and consisted of cisplatin

$152\left(75 \mathrm{mg} / \mathrm{m}^{2}\right.$; 4-hour infusion) on day 1 and 5 -fluorouracil $\left(1000 \mathrm{mg} / \mathrm{m}^{2}\right.$; continuous infusion $)$ on

153 days $1-4$ every 4 weeks. For patients with creatinine clearance $<60 \mathrm{~mL} / \mathrm{min}$, carboplatin was

154 used instead of eisplatin.

155 The technique above-mentioned was performed according to previously published studies

156 (Chen YHet al., 2017; Chen et al., 2017; Chen et al., 2018). 
159 Comparisons between the groups were performed using the chi-square test for categorical

160 variable data. A Cox regression model was used for univariate and multivariable analyses, and

161 the hazard ratio (HR) and 95 confidence interval (CI) were computed with the Cox proportional

162 hazards model. Overall survival (OS) was calculated from the date of diagnosis of the

163 esophageal cancer to the date of death or last contact. The Kaplan-Meier method was used to

164 estimate OS, and the log rank test was performed to evaluate the differences between the groups

165 for univariate analysis. The statistical analyses were performed with the SPSS 19 software

166 package (IBM, Armonk, NY). All of the tests were two-sided tests, and $\mathrm{P}<0.05$ was considered

167 statistically significant.

168

169 Results

Patient characteristics

171 There were 411 locally advanced inoperable ESCC patients who completed CCRT with a

172 curative intent at Kaohsiung Chang Gung Memorial Hospital who were retrospectively

173 investigated in this study, including 63 cervical ESCC patients. All 63 cervical ESCC patients

174 had an Eastern Cooperative Oncology Group performance status $\leq 1$, and these patients consisted 
175 of 61 male patients and 2 female patients with a median age of 58 years (range, 37-80 years).

176 The tumor $\mathrm{T}$ status was revealed to be $\mathrm{T} 2$ in 1 patient (1.6\%), T3 in 13 patients $(20.6 \%)$, and $\mathrm{T} 4$

177 in 49 patients (77.8\%). Meanwhile, five patients (7.9\%) were diagnosed as having N0 status, 24

178 patients (38.1\%) were diagnosed as having as having N1 status, 23 patients (36.5\%) were

179 diagnosed as having as having N2 status, and 11 patients (17.5\%) were diagnosed as having as

180 having N3 status. In terms of tumor stage, seven patients $(11.1 \%)$ had stage IIIA, five patients

$181(7.9 \%)$ had stage IIIB, and 51 patients (81.0\%) had stage IIIC. Among the 63 patients, 14

182 patients $(22.3 \%)$ were classified as grade 1,36 patients $(57.1 \%)$ as grade 2 , and 13 patients

$183(20.6 \%)$ as grade 3.

184 Using the propensity score matching method, 63 matched patients of the 348 thoracic ESCC

185 patients were identified to compare to the 63 cervical ESCC patients. Parameters between these

186 two groups were all matched without statistical difference, including tumor age, gender, T status,

187 N status, tumor stage and tumor grade. The clinicopathological characteristics of the these

188 cervical and thoracic ESCC patients are shown in Table 1.

189 

studies (Dutta et al., 2011; Feng et al., 2014; Forrest et al., 2003; McMillan, 2013; Miyata et al.,

2011; Wei et al., 2015; Xie et al., 2016; Yodying et al., 2016). A total of 63 thoracic ESCC score matching method. There were no significant differences in the baseline characteristics of

197 these two groups, except for PLR $(\mathrm{P}=0.047)$. The comparison of inflammatory biomarkers in

198 these cervical and thoracic ESCC patients are shown in Table 2. 
2079.5 had worse OS compared to the other 35 patients who had CRP/albumin ratio $<9.5(12.0$

208 months versus 25.3 months, $\mathrm{P}=0.030$, Figure $2 \mathrm{D}$ ). In a multivariable analysis, $\mathrm{PLR} \geq 103$

209 ( $\mathrm{P}=0.010$, HR: $2.66,95 \%$ CI: $1.27-5.58)$ and $\mathrm{mGPS} \geq 1$ ( $\mathrm{P}=0.030, \mathrm{HR}: 2.03,95 \%$ CI: $1.07-3.86)$

210 were the independent prognostic parameters of worse OS.

211 With respect to thoracic ESCC, the univariate analysis showed that NLR $\geq 2.5(\mathrm{P}=0.041)$,

$212 \mathrm{PLR} \geq 103(\mathrm{P}=0.024), \mathrm{CRP}$ value $>10(\mathrm{P}=0.001), \mathrm{mGPS} \geq 1(\mathrm{P}=0.001)$, and $\mathrm{CRP} /$ albumin ratio

$213 \geq 9.5(\mathrm{P}=0.002)$ were still significant predictors of worse OS, similar to the results in the cervical

214 ESCC group. Worse OS (9.0 months versus 14.4 months, $\mathrm{P}=0.038$, Figure $3 \mathrm{~A}$ ) was also found in

215 the 35 patients with $\geq 2.5$ than the other 28 patients with NLR $<2.5$; and patients with PLR $\geq$

216103 had worse OS compared to others with PLR $<103$ (9.0 months versus 10.9 months, $\mathrm{P}=0.022$,

217 Figure 3B). The total of 35 patients who had $\mathrm{mGPS} \geq 1$ had worse OS compared to the other 28

218 patients with mGPS of 0 ( 8.9 months versus 20.0 months, $\mathrm{P}=0.001$, Figure $3 \mathrm{C}$ ); the 28 patients

219 who had CRP/albumin ratio $\geq 9.5$ were found to have worse OS in comparison with the 35

220 patients who had CRP/albumin ratio $<9.5$ (9.0 months versus 15.9 months, $\mathrm{P}=0.002$, Figure 3D).

221 Patients with NLR $\geq 2.5(\mathrm{P}=0.012$, HR: $2.21,95 \%$ CI: 1.19-4.12) and $\mathrm{mGPS} \geq 1(\mathrm{P}<0.001$, HR: 
$2223.13,95 \%$ CI: 1.66-5.88) had worse OS than others with NLR $<2.5$ and mGPS of 0 in the

223 multivariable analysis. These univariate and multivariable survival analyses are shown in Table 3.

\section{Discussion}

226 Cervical ESCC is a small population of all esophageal cancer but is often locally advanced

227 with nearby structures invasion at initial presentation (Yin et al., 1983). Although cervical ESCC

228 only accounts for less than $10 \%$ of all cases, the management of this rare disease is very

229 challenging. In the past, radical surgery with reconstruction was the gold standard of treatment,

230 but the locally advanced status usually increased the difficulty of surgery, resulting in high

231 mortality and morbidities (Grass et al., 2015). In the recently $y_{2}$ growing evidences have

232 demonstrated that CCRT improved overall survival for ESCC patients, and more and more

233 physicians preferred definitive CCRT rather than surgical resection for cervical ESCC patients in

234 clinical practice (Cooper et al., 1999; Pignon et al., 2009). As far as we know, there were limited

235 studies which focused on the outcome of cervical ESCC patients, and the predictive prognostic

236 biomarkers for this group still lack. However, accumulating evidences have revealed that

237 inflammation-based prognostic scores, such as NLR, PLR, mGPS, etc., were associated with 
238 clinical outcome in several cancer types, including esophageal cancer (Feng et al., 2014;

239 Lindenmann et al., 2014; Pinato et al., 2014; Stotz et al., 2013; Templeton et al., 2014; Yodying

240 et al., 2016). Therefore, the current study is designed to determine the role of these

241 inflammation-based biomarkers in cervical ESCC patients.

242 Systemic inflammation plays an important role in the tumorigenesis, and the mechanism is

243 very complicated. It may cause genetic mutations and instability, suppress antitumor immunity,

244 decrease DNA repair function, and promote the formation of microenvironments, contributing to

245 tumor initiation. In addition, it also induces tumor cell invasion, migration, metastasis and

246 angiogenesis, resulting in tumor progression. The inflammatory factors are mainly derived from

247 the secretion of both host and tumor cells, and the systemic reaction to cancer cells, including

248 some chemokines and cytokines, transcription factors, CRP, circulating immunocytes, and so on

249 (Balkwill, 2012; Elinav et al., 2013; Hoesel \& Schmid, 2013; Nimptsch et al., 2015).

250 In the present study, we selected six biomarkers to evaluate the prognosis in the cervical

251 ESCC patients, including NLR, PLR, CRP, albumin, mGPS and CRP/albumin ratio. Neutrophil

252 play an important role in the systemic inflammation, and platelets are a critical source of

253 chemokines/cytokines, and they both promote tumor progression through many different 
254 pathways (Balkwill \& Mantovani, 2001; Grivennikov et al., 2010). NLR and PLR were well

255 examined to predict the prognosis and have been frequently used in clinical practice in several

256 cancer types (Feng et al., 2013; Stotz et al., 2013; Templeton et al., 2014). CRP is a protein of

257 acute phase inflammation and has been reported to be associated with prognosis in esophageal

258 cancer patients (Nozoe et al., 2001). Albumin level is a good tool to evaluate the nutrition status

259 in cancer patients, and malnutrition is strongly correlated with worse treatment response and

260 poor prognosis (Hu et al., 2009). The scoring system of mGPS, reported by McMillan, has been

261 reported to be related to tumor size, lymph node metastasis, degree of tumor invasion, and

262 overall survival in ESCC patients (McMillan, 2008). The CRP/albumin ratio was initially

263 developed to predict clinical outcome and complications in patients with severe medical illness,

264 such as sepsis; after that, it was also indicated to predict prognosis in some cancer patients

265 (Ranzani et al., 2013; Wei et al., 2015). These biomarkers, such as complete blood

266 count/differential count, CRP, and albumin, are easy-to-measure, and most are usually

267 considered as routine pre-treatment tests in clinical practice.

268 The goal of treatment between thoracic and cervical ESCC is a little different. For cervical

269 ESCC patients, definitive CCRT is the more preferred therapeutic modality rather than surgical 
resection due to high mortality and morbidities; therefore, higher radiotherapy dose (66-70 Gy)

271 was planned for these patients. In contrast, the radiotherapy dose for thoracic ESCC patients who

272 received CCRT is around $50 \mathrm{~Gy}$. The different radiotherapy doses may result in different

273 treatment response of CCRT and prognosis; although there were limited evidences to discuss the

274 issue. Therefore, the role of inflammation-based prognostic scores for cervical and thoracic

275 ESCC may be different. In previous studies, these biomarkers were well investigated and defined

276 as prognostic factors in thoracic ESCC patients; however, the crucial role in cervical ESCC

277 patients was unclear (Dutta et al., 2011; Feng et al., 2014; McMillan, 2008; Miyata et al., 2011;

278 Wei et al., 2015; Xie et al., 2016; Yodying et al., 2016). The current study showed that NLR,

279 PLR, CRP, mGPS and CRP/albumin ratio were strongly correlated to poor prognosis in cervical

280 ESCC patients. Moreover, in order to correct for bias, the propensity score matching method was

281 used to select a control group from among the locally advanced thoracic ESCC patients who

282 received curative CCRT according to clinical tumor parameters (TNM stage, grade, sex and age)

283 in our hospital. The prognostic value of these biomarkers in the matched thoracic ESCC patients

284 was similar and compatible to the results in the cervical ESCC group in the univariate and

285 multivariable analyses. 
286 Several studies have revealed that sex is an independent prognostic factor of overall

287 survival in esophageal cancer (Chen et al., 2013; Cheng et al., 2018; Micheli et al., 2009). In

288 Taiwan, the male/female incidence ratio of esophageal cancer was 16.2, and male patients were

289 reported to have significant $t_{2}$ worse prognosis compared to female patients (Cheng et al., 2018). In

290 the current study, there were only 2 female patients and the male/female ratio was 30.5 ; therefore,

291 the effect of sex in the survival analysis may be minimal.

292 There are some limitations in our current study. First, the study only enrolled a small

293 sample size, and all patients were treated at a single institution. Second, the study is a

294 retrospective review, such that there may be selection bias. However, to be best of our

295 knowledge, the current study is the first study to investigate the crucial role of inflammation-

296 based prognostic scores in cervical ESCC patients. Furthermore, it comprises the largest series

297 thus far of locally advanced cervical ESCC patients who underwent curative CCRT, and may be

298 helpful to clarify the situation of this rare ESCC group.

299

300 Conclusions 
301 Our study suggests that the inflammation-based prognostic scores are helpful in clinical

302 practice, and PLR and mGPS may predict the prognosis for locally advanced cervical ESCC

303 patients who receiving curative CCRT.

304

305 Acknowledgements

306 We appreciated the Biostatistics Center, Kaohsiung Chang Gung Memorial Hospital for

307 statistics work.

308

309 References

310 National Department of Health, Republic of China. Cancer Registry Annual Report 2015.

311 Balkwill F, and Mantovani A. 2001. Inflammation and cancer: back to Virchow? Lancet

312 357:539-545. 10.1016/S0140-6736(00)04046-0

313 Balkwill FR. 2012. The chemokine system and cancer. J Pathol 226:148-157. 10.1002/path.3029

314 Chen MF, Yang YH, Lai CH, Chen PC, and Chen WC. 2013. Outcome of patients with

315 esophageal cancer: a nationwide analysis. Ann Surg Oncol 20:3023-3030.

$10.1245 / \mathrm{s} 10434-013-2935-4$ 
317 Chen YH LH, Wang YM, Lo CM, Chou SY, Huang CH, Shih LH, Chen SW and Li SH. 2017.

318 The prognostic significance of celiac lymph node metastasis

319 in patients with locally advanced esophageal squamous cell

320 carcinoma receiving curative concurrent chemoradiotherapy. Oncotarget 8:96190-96202.

321 Chen YH, Lu HI, Chien CY, Lo CM, Wang YM, Chou SY, Su YY, Shih LH, and Li SH. 2017.

Treatment Outcomes of Patients with Locally Advanced Synchronous Esophageal and

323

Head/Neck Squamous Cell Carcinoma Receiving Curative Concurrent

324

Chemoradiotherapy. Sci Rep 7:41785. 10.1038/srep41785

325

Chen YH, Lu HI, Lo CM, Wang YM, Chou SY, Huang CH, Shih LH, Chen SW, and Li SH.

326

2018. The clinical impact of supraclavicular lymph node metastasis in patients with

locally advanced esophageal squamous cell carcinoma receiving curative concurrent

328

chemoradiotherapy. PLoS One 13:e0198800. 10.1371/journal.pone.0198800

329

Cheng YF, Chen HS, Wu SC, Chen HC, Hung WH, Lin CH, and Wang BY. 2018. Esophageal squamous cell carcinoma and prognosis in Taiwan. Cancer Med. 10.1002/cam4.1499

331 Cooper JS, Guo MD, Herskovic A, Macdonald JS, Martenson JA, Jr., Al-Sarraf M, Byhardt R,

Russell AH, Beitler JJ, Spencer S, Asbell SO, Graham MV, and Leichman LL. 1999. 
333 Chemoradiotherapy of locally advanced esophageal cancer: long-term follow-up of a

Dutta S, Crumley AB, Fullarton GM, Horgan PG, and McMillan DC. 2011. Comparison of the prognostic value of tumour- and patient-related factors in patients undergoing potentially curative resection of oesophageal cancer. World J Surg 35:1861-1866. 10.1007/s00268011-1130-7

Edge S BD, Compton CC, Fritz AG, Greene FL and Trotti A. AJCC Cancer Staging Manual. 7th ed. New York: Springer-Verlag; 2010.

342 Elinav E, Nowarski R, Thaiss CA, Hu B, Jin C, and Flavell RA. 2013. Inflammation-induced cancer: crosstalk between tumours, immune cells and microorganisms. Nat Rev Cancer

Feng JF, Huang Y, and Chen QX. 2014. Preoperative platelet lymphocyte ratio (PLR) is superior to neutrophil lymphocyte ratio (NLR) as a predictive factor in patients with esophageal squamous cell carcinoma. World J Surg Oncol 12:58. 10.1186/1477-7819-12-58 
lymphocyte ratio is a useful predictor of postoperative survival in patients with esophageal squamous cell carcinoma. Onco Targets Ther 6:1605-1612.

Forrest LM, McMillan DC, McArdle CS, Angerson WJ, and Dunlop DJ. 2003. Evaluation of cumulative prognostic scores based on the systemic inflammatory response in patients with inoperable non-small-cell lung cancer. Br J Cancer 89:1028-1030. $10.1038 /$ sj.bjc. 6601242

Grass GD, Cooper SL, Armeson K, Garrett-Mayer E, and Sharma A. 2015. Cervical esophageal cancer: a population-based study. Head Neck 37:808-814. 10.1002/hed.23678 cancer. Mol Cancer 12:86. 10.1186/1476-4598-12-86

362 Hu JY, Yi W, Xia YF, Gao J, Liu ZG, and Tao YL. 2009. [Impact of pretherapy body mass 363 index on prognosis of nasopharyngeal carcinoma]. Ai Zheng 28:1043-1048.

364 Lindenmann J, Fink-Neuboeck N, Koesslbacher M, Pichler M, Stojakovic T, Roller RE, Maier A, 
Anegg U, Smolle J, and Smolle-Juettner FM. 2014. The influence of elevated levels of Creactive protein and hypoalbuminemia on survival in patients with advanced inoperable esophageal cancer undergoing palliative treatment. J Surg Oncol 110:645-650. 454:436-444. 10.1038/nature07205

374 McMillan DC. 2013. The systemic inflammation-based Glasgow Prognostic Score: a decade of experience in patients with cancer. Cancer Treat Rev 39:534-540.

377 McMillan DC, Elahi MM, Sattar N, Angerson WJ, Johnstone J, and McArdle CS. 2001. 
381 Micheli A, Ciampichini R, Oberaigner W, Ciccolallo L, de Vries E, Izarzugaza I, Zambon P,

382 Gatta G, De Angelis R, and Group EW. 2009. The advantage of women in cancer 10.1016/j.ejca.2008.11.008 operative chemotherapy followed by surgery for esophageal cancer. Exp Ther Med 2:879885. 10.3892/etm.2011.308

Nimptsch K, Aleksandrova K, Boeing H, Janke J, Lee YA, Jenab M, Bueno-de-Mesquita HB, Jansen EH, Tsilidis KK, Trichopoulou A, Weiderpass E, Wu C, Overvad K, Tjonneland Trichopoulos D, Palli D, Agnoli C, Tumino R, Vineis P, Panico S, Johansson A, Van Guelpen B, Khaw KT, Wareham N, Peeters PH, Quiros JR, Vencesla Garcia A, MolinaMontes E, Dorronsoro M, Chirlaque MD, Barricarte Gurrea A, Key TJ, Duarte-Salles T, Stepien M, Gunter MJ, Riboli E, and Pischon T. 2015. Association of CRP genetic A, Boutron-Ruault MC, Dossus L, Racine A, Kaaks R, Canzian F, Lagiou P, variants with blood concentrations of C-reactive protein and colorectal cancer risk. Int J 
Cancer 136:1181-1192. 10.1002/ijc.29086

398 Nozoe T, Saeki H, and Sugimachi K. 2001. Significance of preoperative elevation of serum Creactive protein as an indicator of prognosis in esophageal carcinoma. Am J Surg 182:197-201.

401 Pignon JP, le Maitre A, Maillard E, Bourhis J, and Group M-NC. 2009. Meta-analysis of and 17,346 patients. Radiother Oncol 92:4-14. 10.1016/j.radonc.2009.04.014 performance of inflammation-based prognostic indices in primary operable non-small cell lung cancer. Br J Cancer 110:1930-1935. 10.1038/bjc.2014.145 protein/albumin ratio predicts 90-day mortality of septic patients. PLoS One 8:e59321. 10.1371/journal.pone.0059321 2011. Elevated preoperative neutrophil:lymphocyte ratio as a predictor of postoperative disease recurrence in esophageal cancer. Ann Surg Oncol 18:3362-3369. 10.1245/s10434- 
414 Stotz M, Gerger A, Eisner F, Szkandera J, Loibner H, Ress AL, Kornprat P, AlZoughbi W, operable and inoperable pancreatic cancer. Br J Cancer 109:416-421. $10.1038 /$ bjc. 2013.332

427 Xie X, Luo KJ, Hu Y, Wang JY, and Chen J. 2016. Prognostic value of preoperative platelet- 
esophageal squamous cell cancer. Dis Esophagus 29:79-85. 10.1111/dote.12296 1983. The results of high-energy electron therapy in carcinoma of the oesophagus compared with telecobalt therapy. Clin Radiol 34:113-116. Lymphocyte Ratio in Oncologic Outcomes of Esophageal Cancer: A Systematic Review and Meta-analysis. Ann Surg Oncol 23:646-654. 10.1245/s10434-015-4869-5 Lee CG, and Lee YC. 2014. Prognostic value of neutrophil-to-lymphocyte ratio in patients treated with concurrent chemoradiotherapy for locally advanced oesophageal 


\section{Table $\mathbf{1}$ (on next page)}

Clinicopathological parameters in 126 locally advanced cervical/thoracic esophageal SCC patients receiving curative CCRT 
Table 1. Clinicopathological parameters in $\mathbf{1 2 6}$ locally advanced cervical/thoracic esophageal SCC patients receiving curative CCRT

\begin{tabular}{|c|c|c|c|}
\hline Characteristics & $\begin{array}{c}\text { \#Cervical esophageal } \\
\text { SCC group }(\mathrm{N}=63)\end{array}$ & $\begin{array}{l}\text { \#Thoracic esophageal } \\
\text { SCC group }(\mathrm{N}=63)\end{array}$ & $\mathrm{P}$ value \\
\hline \multicolumn{4}{|l|}{ Age } \\
\hline$<60$ years & $37(58.7 \%)$ & 37 (58.7\%) & 1.0 \\
\hline$\geq 60$ years & $26(41.3 \%)$ & $26(41.3 \%)$ & \\
\hline
\end{tabular}

Sex

$\begin{array}{lccc}\text { Male } & 61(96.8 \%) & 61(96.8 \%) & 1.0 \\ \text { Female } & 2(3.2 \%) & 2(3.2 \%) & \end{array}$

T status

2

3

4

$\mathrm{N}$ status

0

1

2

3

Stage

IIIA

$7(11.1 \%)$

$5(7.9 \%)$

$51(81.0 \%)$

IIIC

Grade

1

2

3

IIIB
$1(1.6 \%)$

$13(20.6 \%)$

$49(77.8 \%)$

$5(7.9 \%)$

$24(38.1 \%)$

$23(36.5 \%)$

$11(17.5 \%)$

$1(1.6 \%)$

1.0

$13(20.6 \%)$

$49(77.8 \%)$

$24(38.1 \%)$

$23(36.5 \%)$

$11(17.5 \%)$

$7(11.1 \%)$

1.0

$5(7.9 \%)$

$51(81.0 \%)$

$14(22.3 \%) \quad 1.0$

$36(57.1 \%)$

$13(20.6 \%)$

SCC: squamous cell carcinoma; CCRT: concurrent chemoradiotherapy; \#Using propensity score matching method. *Statistically significant. 
Table 2 (on next page)

Comparison of inflammation-based prognostic scores in 126 locally advanced cervical/thoracic esophageal SCC patients receiving curative CCRT 
Table 2. Comparison of inflammation-based prognostic scores in 126 locally advanced cervical/thoracic esophageal SCC patients receiving curative CCRT

\begin{tabular}{lccc}
\hline Characteristics & \#Cervical esophageal & \#Thoracic & \\
& SCC group $(\mathrm{N}=63)$ & $\begin{array}{c}\text { esophageal SCC } \\
\text { group }(\mathrm{N}=63)\end{array}$ & P value \\
\hline
\end{tabular}

Neutrophil lymphocyte ratio

$\begin{array}{llll}<2.5 & 28(44 \%) & 19(30 \%) & 0.097 \\ \geq 2.5 & 35(56 \%) & 44(70 \%) & \end{array}$

Platelet lymphocyte ratio

$\begin{array}{llll}<103 & 42(67 \%) & 31(49 \%) & 0.047^{*} \\ \geq 103 & 21(33 \%) & 32(51 \%) & \end{array}$

Albumin
$<3.5$
$9(14 \%)$
$12(19 \%)$
0.473
$\geq 3.5$
$54(86 \%)$
$51(81 \%)$

CRP

$\begin{array}{llll}\leq 10 & 28(44 \%) & 21(33 \%) & 0.201 \\ >10 & 35(56 \%) & 42(67 \%) & \end{array}$

mGPS
0
$28(44 \%)$
$21(33 \%)$
0.201
$1+2$
$35(56 \%)$
$42(67 \%)$

$\mathrm{CRP} /$ Albumin ratio

$\begin{array}{llll}<9.5 & 35(56 \%) & 34(54 \%) & 0.858 \\ \geq 9.5 & 28(44 \%) & 29(46 \%) & \end{array}$

SCC: squamous cell carcinoma; CCRT: concurrent chemoradiotherapy; CRP: Creactive protein; mGPS: modified Glasgow Prognostic Score. \#Using propensity score matching method. *Statistically significant. 


\section{Table 3 (on next page)}

Univariate and multivariable analysis of overall survival in in 126 locally advanced cervical/thoracic esophageal SCC patients receiving curative CCRT 
Table 3. Univariate and multivariable analysis of overall survival in in $\mathbf{1 2 6}$ locally advanced cervical/thoracic esophageal SCC patients receiving curative CCRT

\begin{tabular}{|c|c|c|c|c|}
\hline \multirow{3}{*}{ Characteristics } & \multicolumn{4}{|c|}{ \#Cervical esophageal SCC group } \\
\hline & \multicolumn{2}{|c|}{ Univariate analysis } & \multicolumn{2}{|c|}{ Multivariable analysis } \\
\hline & $\operatorname{HR}(95 \% \mathrm{CI})$ & $\mathrm{P}$ value & $\mathrm{HR}(95 \% \mathrm{CI})$ & $\mathrm{P}$ value \\
\hline Neutrophil lymphocyte ratio $\geq 2.5$ & $2.19(1.14-4.20)$ & $0.019^{*}$ & & \\
\hline Platelet lymphocyte ratio $\geq 103$ & $2.51(1.22-5.17)$ & $0.013^{*}$ & $2.66(1.27-5.58)$ & $0.010^{*}$ \\
\hline Albumin $\geq 3.5$ & $0.70(0.31-1.61)$ & 0.404 & & \\
\hline $\mathrm{CRP}>10$ & $1.95(1.03-3.69)$ & $0.037 *$ & & \\
\hline $\mathrm{mGPS} \geq 1$ & $1.95(1.03-3.69)$ & $0.037^{*}$ & $2.03(1.07-3.86)$ & $0.030^{*}$ \\
\hline \multirow[t]{2}{*}{$\mathrm{CRP} /$ Albumin ratio $\geq 9.5$} & $1.96(1.06-3.64)$ & $0.033^{*}$ & & \\
\hline & \multicolumn{4}{|c|}{ \#Thoracic esophageal SCC group } \\
\hline \multirow[t]{2}{*}{ Characteristics } & \multicolumn{2}{|c|}{ Univariate analysis } & \multicolumn{2}{|c|}{ Multivariable analysis } \\
\hline & $\operatorname{HR}(95 \% \mathrm{CI})$ & $\mathrm{P}$ value & $\mathrm{HR}(95 \% \mathrm{CI})$ & $\mathrm{P}$ value \\
\hline Neutrophil lymphocyte ratio $\geq 2.5$ & $1.87(1.03-3.42)$ & $0.041^{*}$ & $2.21(1.19-4.12)$ & $0.012 *$ \\
\hline Platelet lymphocyte ratio $\geq 103$ & $1.89(1.09-3.28)$ & $0.024 *$ & & \\
\hline Albumin $\geq 3.5$ & $0.68(0.35-1.32)$ & 0.246 & & \\
\hline $\mathrm{CRP}>10$ & $2.78(1.50-5.15)$ & $0.001 *$ & & \\
\hline $\mathrm{mGPS} \geq 1$ & $2.78(1.50-5.15)$ & $0.001 *$ & $3.13(1.66-5.88)$ & $<0.001^{*}$ \\
\hline $\mathrm{CRP} /$ Albumin ratio $\geq 9.5$ & $2.42(1.38-3.24)$ & $0.002 *$ & & \\
\hline
\end{tabular}


CCRT: concurrent chemoradiotherapy; HR: hazard ratio; CI: confidence interval; CRP: C-reactive protein; mGPS: modified Glasgow Prognostic Score. \#Using propensity score matching method. *Statistically significant. 


\section{Figure 1}

Algorithm for identifying locally advanced cervical and thoracic esophageal squamous cell carcinoma (ESCC) patients

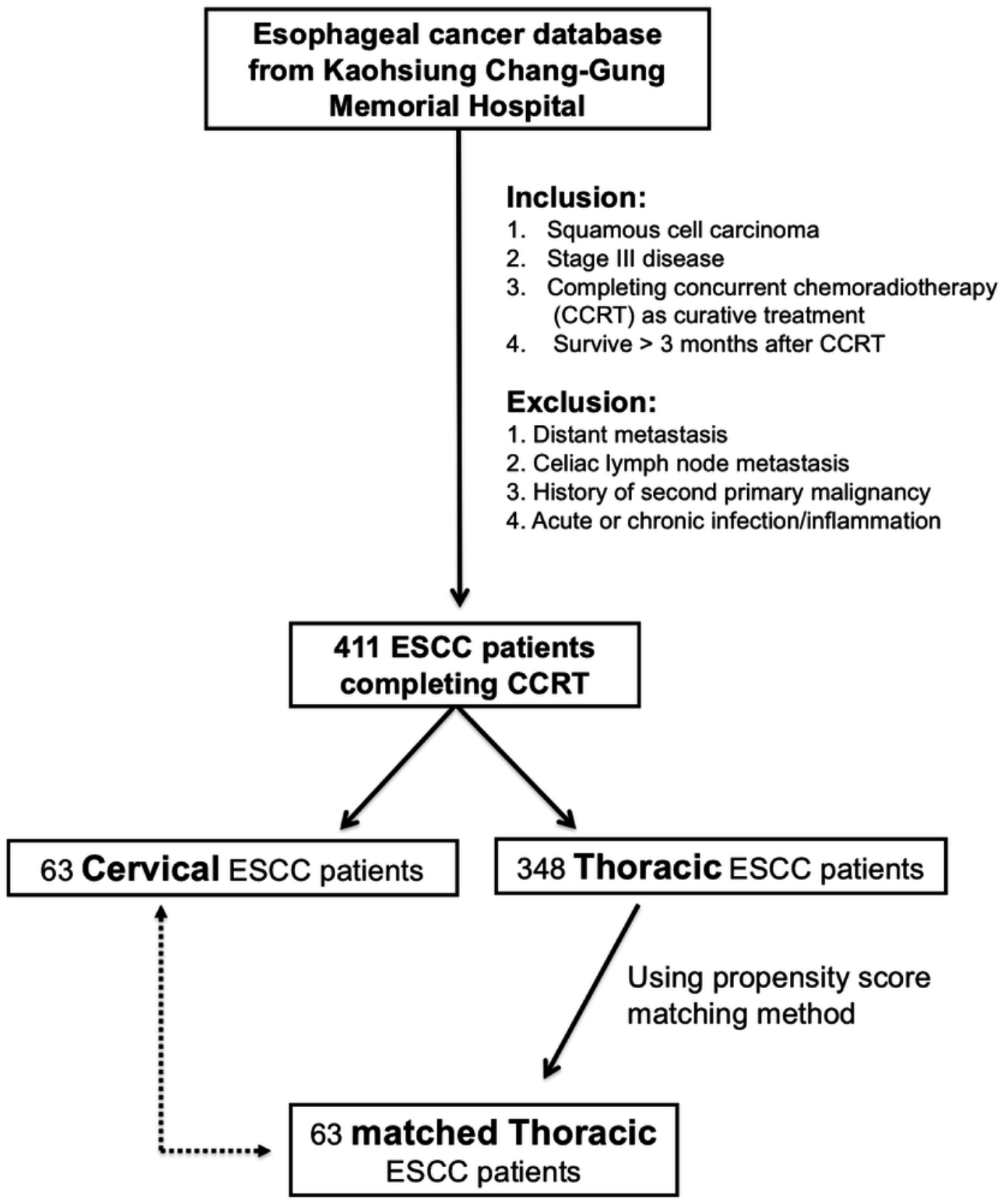


Figure 2

Comparison of overall survival curves of cervical esophageal squamous cell carcinoma patients according to different inflammation-based prognostic scores.

(A) Neutrophil lymphocyte ratio (NLR). (B) Platelet lymphocyte ratio (PLR) (C) Modified Glasgow prognostic score (mGPS). (D) CRP/albumin ratio.

(A)

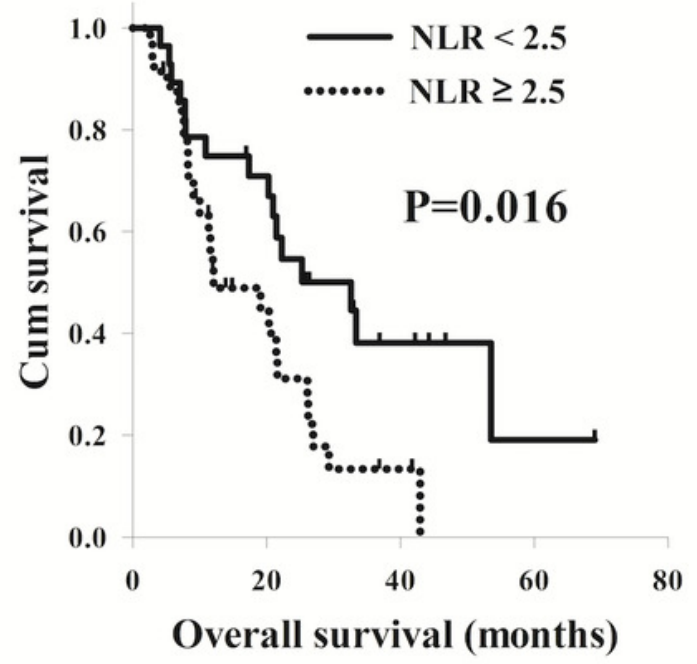

(C)

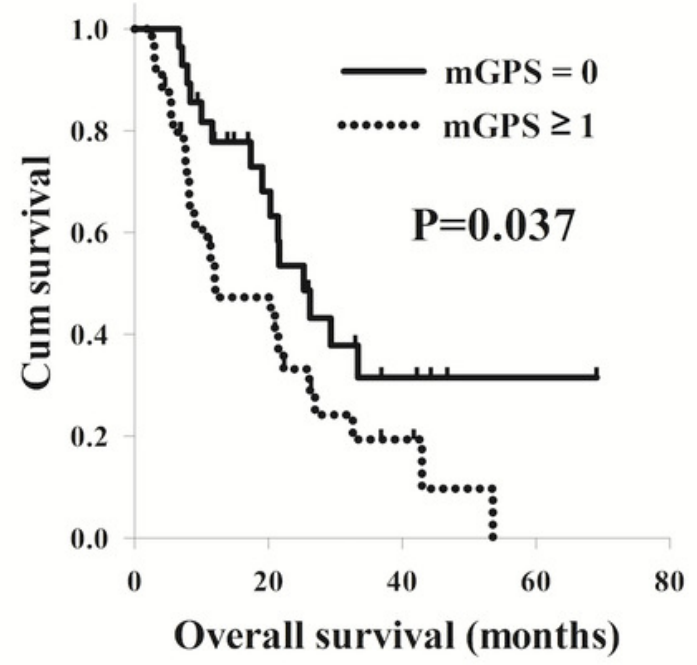

(B)

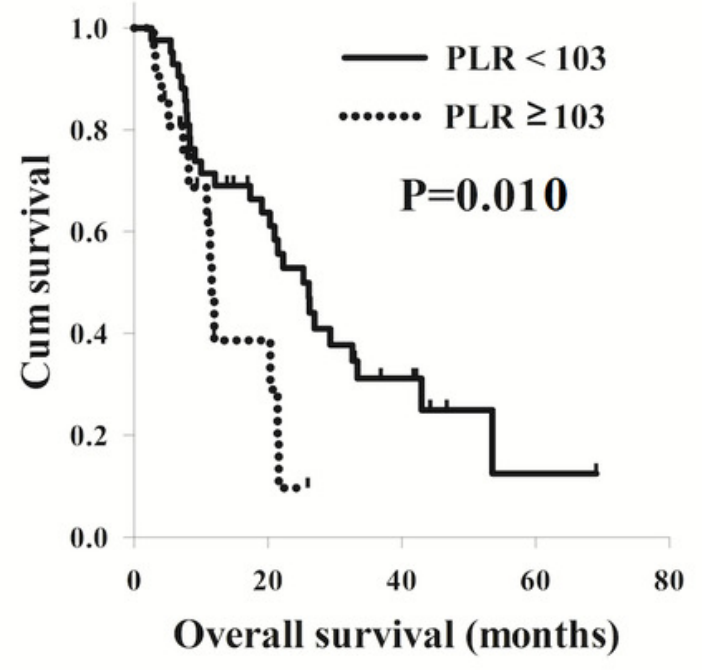

(D)

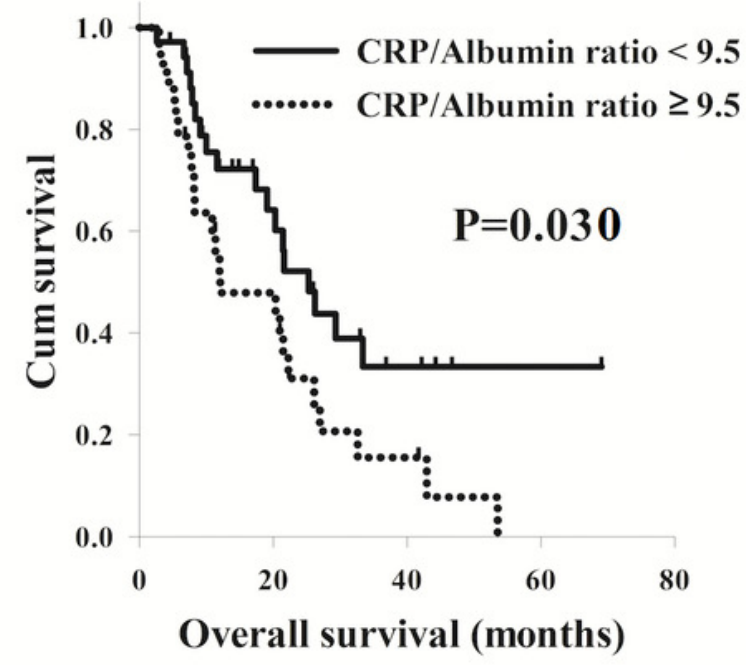


Figure 3

Comparison of overall survival curves of thoracic esophageal squamous cell carcinoma patients according to different inflammation-based prognostic scores.

(A) Neutrophil lymphocyte ratio (NLR). (B) Platelet lymphocyte ratio (PLR) (C) Modified Glasgow prognostic score (mGPS). (D) CRP/albumin ratio.

(A)

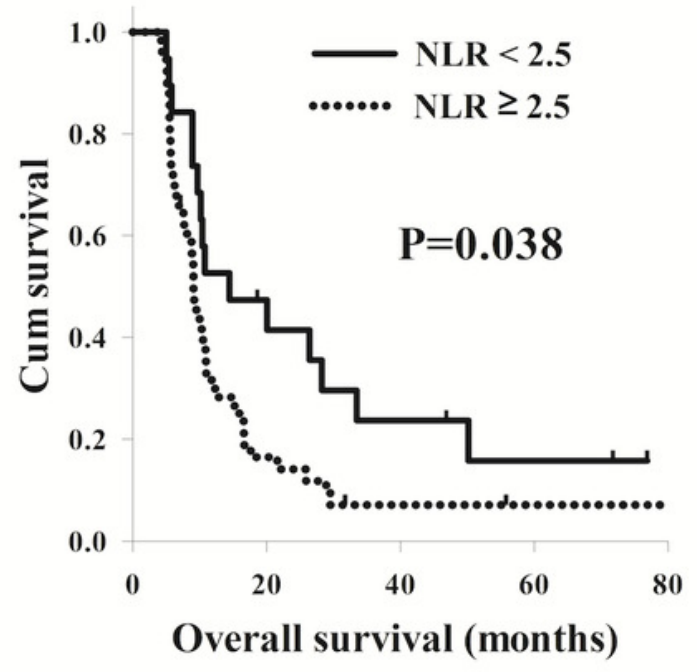

(C)

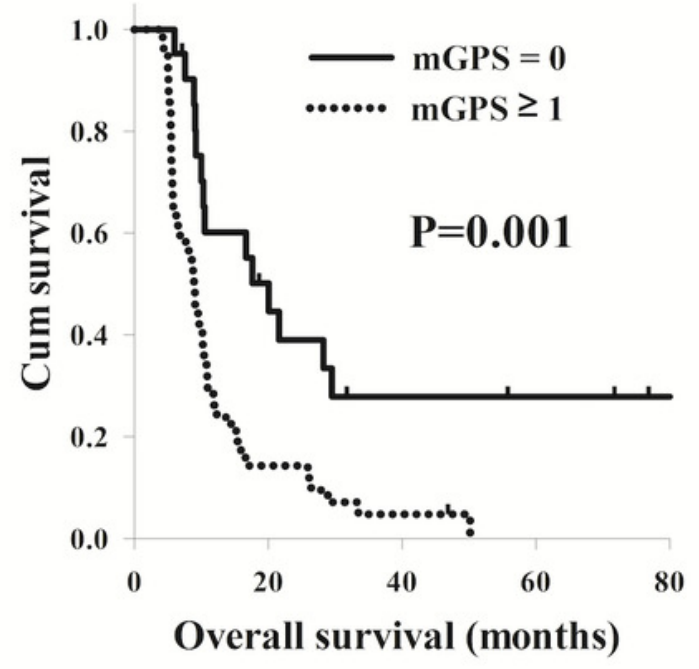

(B)

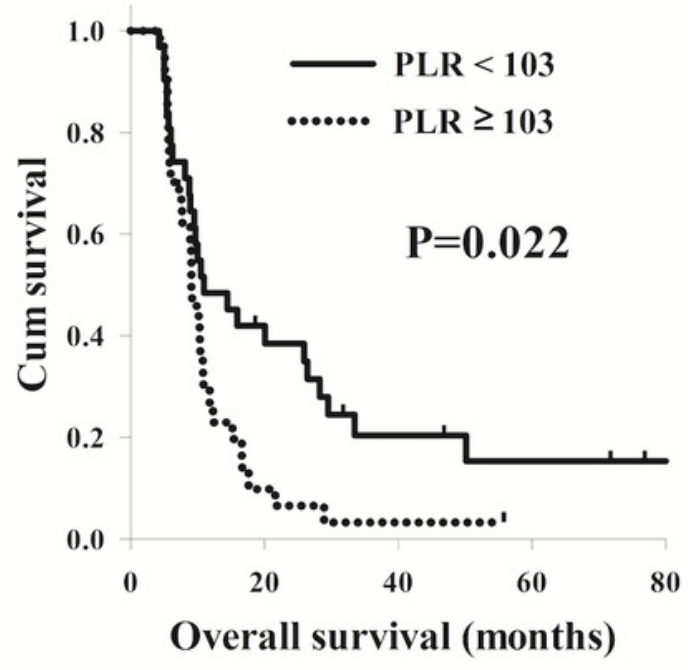

(D)

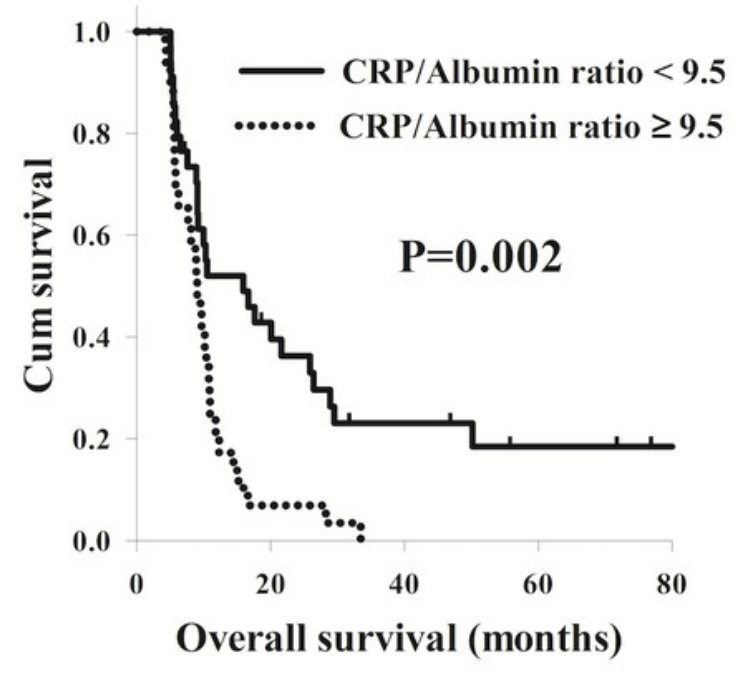

